\title{
Understanding the complexities of collecting and using PRO data in a primary care context
}

\author{
Joanne Greenhalgh (1) 1,2
}

${ }^{1}$ School of Sociology and Social Policy, University of Leeds, Leeds, UK

${ }^{2}$ President Elect, International Society for Quality of Life Research (ISOQOL)

\section{Correspondence to}

Professor Joanne Greenhalgh, School of Sociology and Social Policy, University of Leeds, Leeds LS2 9JT, UK;

j.greenhalgh@leeds.ac.uk

Accepted 29 July 2021

\section{Linked}

- http://dx.doi.org/10.1136/ bmjqs-2020-012206

\section{Check for updates}

(C) Author(s) (or their employer(s)) 2021. No commercial re-use. See rights and permissions. Published by BMJ.

To cite: Greenhalgh J. BMJ Qual Saf Epub ahead of print: [please include Day Month Year]. doi:10.1136/ bmjqs-2021-013315
Patient-reported outcomes (PROs) were originally introduced to primary care as screening instruments to assist primary care practitioners to diagnose and manage depression, on the assumption that standardised tools offered a more systematic and comprehensive assessment of symptoms. ${ }^{12}$ Since then, in both England and the USA, for instance, primary care has seen a move towards integrated care systems with collaboration across primary and secondary care and social services with the aim of better supporting patients to self-manage long-term conditions. ${ }^{3}$ In this context, PROs are envisaged to enable practitioners to identify patients' problems and open up a discussion between the patient and the practitioner on how best to address them. ${ }^{4}$ The electronic capture and feedback of PROs to both clinicians and patients also offer opportunities for patients to track their own symptoms to support self-management. ${ }^{5}$ At the same time, PROs are advocated as a means of monitoring the quality of patient care $^{67}$ and their use has been financially incentivised in some countries through pay-for-performance initiatives. ${ }^{8} 9$ The 'measure once, cut twice' premise of using the same PRO data for multiple uses ${ }^{10} 11$ is appealing but recent reviews of the literature also identified a number of challenges to this approach in practice. ${ }^{12} 13$ There is a need for evidence to demonstrate how PROs can successfully support patient care in primary care and to disentangle and understand the complex and interacting tensions that characterise the reality of work in this context. Such evidence is unlikely to arise from randomised controlled trials that typically adopt a 'black box' approach to studying complexity ${ }^{14}$ but instead from observational studies of the real-world use of PROs in clinical practice.
Against this background, Mou et al's study $^{15}$ in this issue of BMJ Quality \& Safety exploring primary care practitioners' experiences of implementing a wide range of PROs for screening, monitoring and regulatory requirements in a large US health system is a welcome addition to this evidence base. Nineteen primary care clinics serving over 200000 patients administered a questionnaire at patient annual reviews and to new patients. The questionnaires, which were part of a 'Primary Care Screening Bundle', were available for patients to complete electronically prior to the clinic visit and were integrated into the electronic patient record. As of 2019, 74\% of eligible patients had completed screening. Mou et al ${ }^{15}$ surveyed $117 / 172$ primary care practitioners (68\% response rate) and interviewed a purposive sample of 20 primary care practitioners to understand their experiences of using PRO information from the Primary Care Screening Bundle in primary care. Here I consider how their findings shed light on the realities of implementing and using PROs in a primary care context.

Mou et $a l^{15}$ found that use of PROs supported clinical decision-making by highlighting patient issues that may have gone unnoticed by primary care practitioners, a finding that is echoed in reviews of the literature ${ }^{12} 16$ and in recent studies. ${ }^{17} 18$ They also report that PROs improved clinic efficiency, largely by ensuring that items required for regulatory or accreditation purposes were already completed before the consultation. However, Mou et $a l^{15}$ noted that some primary care practitioners did not look at PRO data at all and others felt PRO data did not influence patient management. Herein lies a tension in the collection of PROs for multiple purposes; 
previous reviews have identified that general practitioners felt data required for regulatory or performance management purposes did not always reflect what was medically important in managing patients and so were reluctant to trust or act on these data. ${ }^{12} 13$ Recent studies also suggest that patients question the value of PROs if they are perceived to be a 'tick box' exercise and clinicians do not discuss the results with them. ${ }^{19}$ These findings resonate with learning points from analogous tools such as computerised templates or checklists for the review of long-term conditions ${ }^{2021}$ and structured needs assessments ${ }^{22}$ where there is a risk that the structured nature of the tool leads practitioners to prioritise a biomedical agenda and limits the scope for patients to elaborate their concerns.

Mou et al's ${ }^{15}$ study provides further insight into these complexities. They observed that some primary care clinicians felt the questions they had to ask for regulatory purposes were often tangential to or out of kilter with the clinical reason for the patients' visit. They also found that patients sometimes could not understand why they were being asked these questions or were concerned that data were being used to 'profile' them. Others have also found that some primary care practitioners felt 'bamboozling' patients with lots of questions to complete a PRO was not a clinical priority and that PROs do not replace a faceto-face discussion with patients. ${ }^{17} 23$ These findings highlight that primary care consultations involve more than just information exchange but are sites of relationship building and management ${ }^{24}$; primary care practitioners' use of PROs is entangled with this ongoing accomplishment and in turn shapes how PRO data are understood and used. ${ }^{25}{ }^{26}$ Recent studies in other contexts have shown that using PROs in a 'relational way', whereby practitioners sit with patients while they complete the PRO and probe their answers, can open up opportunities for dialogue and strengthen practitioner-patient relationships. ${ }^{27}$

Mou et al's ${ }^{15}$ findings also revealed challenges to integrating the collection and use of PRO data into the clinic workflow. In Mou et al's ${ }^{15}$ study, patients could complete their questionnaires online prior to the clinic visit or on a tablet in the waiting room and data were then available in real time through the electronic health record (EHR) during the clinic visit. However, some primary care practitioners reported that there was not enough time to complete the items in the waiting room resulting in incomplete data and that front desk staff felt collection of PRO data in the waiting room made the clinic less efficient. Primary care practitioners also reported difficulties finding and understanding PRO data in the EHR due to poor formatting and presentation. Similar challenges have been observed elsewhere ${ }^{17} 28$ and others have noted that EHRs are often not designed to incorporate PRO data. ${ }^{29}$ Guidance exists to support the integration of PRO data into EHRs, ${ }^{30}$ the optimal presentation of PRO data to assist their interpretation ${ }^{31}$ and the training of practitioners in the use of PRO data. ${ }^{32}$ While undoubtedly useful, such guidance is unlikely to resolve all of the complexities that underlie the work of collecting and using PRO data to support patient care. These complexities often require an understanding of professional and organisational practices and routines and how these shape and are shaped by technology. ${ }^{33}$ Thus, it is worth briefly considering how scholars from science and technology studies have understood PRO and EHR data.

These scholars have noted how patient data contained in EHRs function as a 'data double' of the patient. ${ }^{3435}$ This creates a separation between locally produced patient narratives, elicited during interactions between patients and primary care practitioners within consultations, and PRO data in EHRs. ${ }^{36}$ Langstrup $^{37}$ argues that there may be tensions between PRO data and patients' own accounts of their problems that clinicians must actively resolve. Her study showed that PRO data may illuminate, act as a substitute for or even inhibit the patient's own narrative. ${ }^{37}$ Other studies have also found that primary care practitioners do not always trust the 'data double' produced by PROs; for example, they have expressed concerns that patients may either under-report or over-report problems when completing PROs or that PRO items 'put ideas into [patients'] heads' about how they should feel. ${ }^{17}$ However, a review found that while some patients felt PROs did not always capture the complexity of their problems, most studies reported that PRO completion leads patients to reflect on their condition and, in turn, this helps to clarify how they feel and can give patients permission to raise issues with practitioners. ${ }^{25}$ Others have found a poor agreement between PRO data and EHRs-primary care practitioners did not record diagnostic codes based on PRO data unless the patient also reported it was a problem. ${ }^{29}$ This suggests primary care practitioners do not always accept the content validity of PROs, and the patient's own narrative is required as verification. We might question whether practitioners' scepticism of PRO data reflects a 'doctor knows best' mindset; these sorts of objections are not limited to primary care but have been found in other settings. ${ }^{38}$ However, they might also reflect practitioners' struggles to reconcile different 'versions' of the patient being presented to them.

These findings also signify the different mechanisms through which PROs can support patient care, either by acting as a 'test result' to raise practitioners' awareness of patients' problems or through enabling patients to better identify these issues and raise them with practitioners. ${ }^{25}$ This dichotomy could also explain the conflicting accounts from primary care practitioners found by Mou et $a l^{15}$ and others ${ }^{17}$ regarding whether PRO data improve the efficiency of the consultation or informed clinical management. If PRO data act like a test result that can be taken at face value by practitioners, then it 
may serve to pinpoint patients' problems more efficiently. However, if PRO data either require further corroboration from the patient and, in turn, reveal a mismatch between the PRO data and the patient's account or open up issues that practitioners feel are beyond their remit to address or are ill equipped to manage, then this may lengthen consultations significantly. Primary care practitioners in Mou et al's ${ }^{15}$ study and others ${ }^{17}$ often draw attention to the material reality of the time pressure of a primary care context, which, given the complexities of interpreting these data, is a very real constraint on how practitioners engage with PRO data.

Several authors have provided valuable frameworks and lessons learnt to support the implementation of PROs into clinical practice. ${ }^{39-41}$ Addressing the technical design of EHRs, improving the presentation of PRO data and training to increase practitioners' understanding of PROs are all important. However, the fundamental tension between the need to explore patients' experiences in sufficient detail and practitioners feeling they lack the time, remit or resources to do so may be less easy to resolve. Furthermore, if PRO data are to be successfully used for multiple purposes, this will also add complexity in data collection, clinic workflow and interpretation of data. Other studies have shown that the use of outcome measures and interpretation of outcome data requires considerable practitioner tacit knowledge, which develops as practitioners gain experience of using these measures in practice. ${ }^{42}$ This suggests that in moving forward we need to understand and support the processes through which PRO use becomes routine and is embedded within professional and organisational norms. ${ }^{43}$ As such, there is much to be gained from the use of implementation science frameworks and theories to facilitate the integration of PRO data into clinical practice to support patient care. ${ }^{44}$ As others have noted, ${ }^{45} 46$ addressing the 'barriers and facilitators' to implementing complex health interventions does not rely on the description of these 'barriers and facilitators' alone but requires addressing the system strains and complexities underlying their use in practice.

\section{Twitter Joanne Greenhalgh @Greenhalgh_Jo}

Acknowledgements This paper is dedicated to my dad, Stan Greenhalgh, who died during the time I was writing it. I would like to thank Perla Marang-van de Mheen and John Browne for their patience, support and constructive comments on an earlier draft of this paper.

Funding The authors have not declared a specific grant for this research from any funding agency in the public, commercial or not-for-profit sectors.

Disclaimer The paper represents the author's views and not necessarily those of ISOQOL.

Competing interests None declared.

Patient consent for publication Not required.
Provenance and peer review Commissioned; internally peer reviewed.

ORCID ID

Joanne Greenhalgh http://orcid.org/0000-0003-2189-8879

\section{REFERENCES}

1 Kendrick T, El-Gohary M, Stuart B, et al. Routine use of patient reported outcome measures (PROMs) for improving treatment of common mental health disorders in adults. Cochrane Database Syst Rev 2016;7:CD011119.

2 Gilbody SM, House AO, Sheldon TA. Routinely administered questionnaires for depression and anxiety: systematic review. BMJ 2001;322:406-9.

3 Baltaxe E, Czypionka T, Kraus M, et al. Digital health transformation of integrated care in Europe: Overarching analysis of 17 integrated care programs. J Med Internet Res 2019;21:e14956.

4 Santana M-J, Feeny D. Framework to assess the effects of using patient-reported outcome measures in chronic care management. Qual Life Res 2014;23:1505-13.

5 Holmes MM, Stanescu S, Bishop FL. The use of measurement systems to support patient self-management of long-term conditions: an overview of opportunities and challenges. Patient Relat Outcome Meas 2019;10:385-94.

6 Murphy M, Salisbury C, Hollinghurst S. Can the outcome of primary care be measured by a patient reported outcome measure? Br J Gen Pract 2014;64:647-8.

7 Keller S, Dy S, Wilson R, et al. Selecting patient-reported outcome measures to contribute to primary care performance measurement: a mixed methods approach. J Gen Intern Med 2020;35:2687-97.

8 Kendrick T, Moore M, Leydon G. Performance indicators for depression management in UK primary care: quantitative and qualitative evaluation of the use of depression symptom questionnaire PROMs. J Ment Health Policy Econ 2014;17:S10-11.

9 Squitieri L, Bozic KJ, Pusic AL. The role of patient-reported outcome measures in value-based payment reform. Value Health 2017;20:834-6.

10 Wu AW, Kharrazi H, Boulware LE, et al. Measure once, cut twice--adding patient-reported outcome measures to the electronic health record for comparative effectiveness research. J Clin Epidemiol 2013;66:S12-20.

11 Van Der Wees PJ, Nijhuis-Van Der Sanden MWG, Ayanian $\mathrm{JZ}$, et al. Integrating the use of patient-reported outcomes for both clinical practice and performance measurement: views of experts from 3 countries. Milbank Q 2014;92:754-75.

12 Greenhalgh J, Dalkin S, Gooding K. Functionality and feedback: a realist synthesis of the collation, interpretation and utilisation of patient-reported outcome measures data to improve patient care. Health Serv Deliv Res 2016;5. doi: 10.3310/hsdr05020

13 Foster A, Croot L, Brazier J, et al. The facilitators and barriers to implementing patient reported outcome measures in organisations delivering health related services: a systematic review of reviews. J Patient Rep Outcomes 2018;2:46.

14 Pawson $\mathrm{R}$. The science of evaluation: a realist manifesto. London: Sage, 2013.

15 Mou D, Horn DM, Heng M, et al. Primary care physician's (PCP) perceived value of patient-reported outcomes (pros) in clinical practice: a mixed methods study. BMJ Qual Saf 2021:bmjqs-2020-012206. 
16 Boyce MB, Browne JP, Greenhalgh J. The experiences of professionals with using information from patient-reported outcome measures to improve the quality of healthcare: a systematic review of qualitative research. BMJ Qual Saf 2014;23:508-18.

17 Litchfield I, Greenfield S, Turner GM, et al. Implementing PROMs in routine clinical care: a qualitative exploration of GP perspectives. BJGP Open 2021;5. doi:10.3399/ bjgpopen20X101135. [Epub ahead of print: 23 Feb 2021].

18 Porter I, Davey A, Gangannagaripalli J, et al. Integrating patient reported outcome measures (PROMs) into routine nurse-led primary care for patients with multimorbidity: a feasibility and acceptability study. Health Qual Life Outcomes 2021;19:133.

19 Talib TL, DeChant P, Kean J, et al. A qualitative study of patients' perceptions of the utility of patient-reported outcome measures of symptoms in primary care clinics. Qual Life Res 2018;27:3157-66.

20 Chew-Graham CA, Hunter C, Langer S, et al. How QOF is shaping primary care review consultations: a longitudinal qualitative study. BMC Fam Pract 2013;14:103.

21 Rhodes P, Langdon M, Rowley E, et al. What does the use of a computerized checklist mean for patient-centered care? the example of a routine diabetes review. Qual Health Res 2006;16:353-76.

22 Cowley S, Mitcheson J, Houston AM. Structuring health needs assessments: the medicalisation of health visiting. Sociol Health Illn 2004;26:503-26.

23 Leydon GM, Dowrick CF, McBride AS, et al. Questionnaire severity measures for depression: a threat to the doctor-patient relationship? Br J Gen Pract 2011;61:117-23.

24 Blakeman T, Bower P, Reeves D, et al. Bringing selfmanagement into clinical view: a qualitative study of long-term condition management in primary care consultations. Chronic Illn 2010;6:136-50.

25 Greenhalgh J, Gooding K, Gibbons E, et al. How do patient reported outcome measures (PROMs) support clinician-patient communication and patient care? A realist synthesis. J Patient Rep Outcomes 2018;2:42.

26 Brewster L, Tarrant C, Willars J, et al. Measurement of harms in community care: a qualitative study of use of the NHS safety thermometer. BMJ Qual Saf 2018;27:625-32.

27 Krawczyk M, Sawatzky R. Relational use of an electronic quality of life and practice support system in hospital palliative consult care: a pilot study. Palliat Support Care 2018:1-6.

28 Mitchell C, Dwyer R, Hagan T, et al. Impact of the QOF and the NICE guideline in the diagnosis and management of depression: a qualitative study. Br J Gen Pract 2011;61:e279-89.

29 Barr PJ, Berry SA, Gozansky WS, et al. No date for the PROM: the association between patient-reported health events and clinical coding in primary care. J Patient Rep Outcomes 2020;4:17.

30 Gensheimer SG, Wu AW, Snyder CF, et al. Oh, the places we'll go: patient-reported outcomes and electronic health records. Patient 2018;11:591-8.
31 Snyder C, Smith K, Holzner B, et al. Making a picture worth a thousand numbers: recommendations for graphically displaying patient-reported outcomes data. Qual Life Res 2019;28:345-56.

32 Santana MJ, Haverman L, Absolom K, et al. Training clinicians in how to use patient-reported outcome measures in routine clinical practice. Qual Life Res 2015;24:1707-18.

33 Berg M. Patient care information systems and health care work: a sociotechnical approach. Int J Med Inform 1999;55:87-101.

34 Berg M, Bowker G. The multiple bodies of the medical record. Sociol Q 1997;38:513-37.

35 Lupton D. M-health and health promotion: the digital cyborg and surveillance Society. Soc Theory Health 2012;10:229-44.

36 Haggerty KD, Ericson RV. The surveillant assemblage. Br J Sociol 2000;51:605-22.

37 Langstrup A. "We can still talk to the patient"' Negotiating the narrative power of patient reported data. 7th International Conference on Infrastructures in Healthcare, 2019.

38 Boyce MB, Browne JP, Greenhalgh J. Surgeon's experiences of receiving peer benchmarked feedback using patientreported outcome measures: a qualitative study. Implement Sci 2014;9:84.

39 Porter I, Gonçalves-Bradley D, Ricci-Cabello I, et al. Framework and guidance for implementing patient-reported outcomes in clinical practice: evidence, challenges and opportunities. J Comp Eff Res 2016;5:507-19.

40 Nordan L, Blanchfield L, Niazi S, et al. Implementing electronic patient-reported outcomes measurements: challenges and success factors. BMJ Qual Saf 2018;27:852-6.

41 Snyder CF, Aaronson NK, Choucair AK, et al. Implementing patient-reported outcomes assessment in clinical practice: a review of the options and considerations. Qual Life Res 2012;21:1305-14.

42 Greenhalgh J, Flynn R, Long AF, et al. Tacit and encoded knowledge in the use of standardised outcome measures in multidisciplinary team decision making: a case study of inpatient neurorehabilitation. Soc Sci Med 2008;67:183-94.

43 May C, Finch T, Implementing FT. Implementing, embedding, and integrating practices: an outline of normalization process theory. Sociology 2009;43:535-54.

44 Stover AM, Haverman L, van Oers HA, et al. Using an implementation science approach to implement and evaluate patient-reported outcome measures (PROM) initiatives in routine care settings. Qual Life Res 2020. doi:10.1007/s11136020-02564-9. [Epub ahead of print: 10 Jul 2020].

45 Brennan C, Greenhalgh J, Pawson R. Guidance on guidelines: understanding the evidence on the uptake of health care guidelines. J Eval Clin Pract 2018;24:105-16.

46 Checkland K, Harrison S, Marshall M. Is the metaphor of 'barriers to change' useful in understanding implementation? Evidence from general medical practice. J Health Serv Res Policy 2007;12:95-100. 